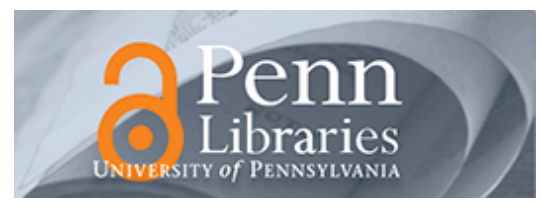

University of Pennsylvania ScholarlyCommons

$5-27-2017$

\title{
Quasi-Static and Dynamic Mismatch for Door Opening and Stair Climbing With a Legged Robot
}

\author{
T. Turner Topping \\ ttopping@seas.upenn.edu \\ Gavin Kenneally \\ University of Pennsylvania, gake@seas.upenn.edu \\ Daniel E. Koditschek \\ University of Pennsylvania, kod@seas.upenn.edu
}

Follow this and additional works at: https://repository.upenn.edu/ese_papers

Part of the Electrical and Computer Engineering Commons, and the Systems Engineering Commons

\section{Recommended Citation}

T. Turner Topping, Gavin Kenneally, and Daniel E. Koditschek, "Quasi-Static and Dynamic Mismatch for Door Opening and Stair Climbing With a Legged Robot", . May 2017.

This paper is posted at ScholarlyCommons. https://repository.upenn.edu/ese_papers/791

For more information, please contact repository@pobox.upenn.edu. 


\title{
Quasi-Static and Dynamic Mismatch for Door Opening and Stair Climbing With a Legged Robot
}

\author{
Abstract \\ This paper contributes to quantifying the notion of robotic fitness by developing a set of necessary \\ conditions that determine whether a small quadruped has the ability to open a class of doors or climb a \\ class of stairs using only quasi-static maneuvers. After verifying that several such machines from the \\ recent robotics literature are mismatched in this sense to the common human scale environment, we \\ present empirical workarounds for the Minitaur quadrupedal platform that enable it to leap up, force the \\ door handle and push through the door, as well as bound up the stairs, thereby accomplishing through \\ dynamical maneuvers otherwise (i.e., quasi-statically) achievable tasks. \\ For more information: Kod*lab \\ Disciplines \\ Electrical and Computer Engineering | Engineering | Systems Engineering
}




\title{
Quasi-Static and Dynamic Mismatch for Door Opening and Stair Climbing With a Legged Robot
}

\author{
T. Turner Topping ${ }^{1}$, Gavin Kenneally ${ }^{2}$, and D. E. Koditschek ${ }^{1}$
}

\begin{abstract}
This paper contributes to quantifying the notion of robotic fitness by developing a set of necessary conditions that determine whether a small quadruped has the ability to open a class of doors or climb a class of stairs using only quasi-static maneuvers. After verifying that several such machines from the recent robotics literature are mismatched in this sense to the common human scale environment, we present empirical workarounds for the Minitaur quadrupedal platform that enable it to leap up, force the door handle and push through the door, as well as bound up the stairs, thereby accomplishing through dynamical maneuvers otherwise (i.e., quasi-statically) unachievable tasks.
\end{abstract}

\section{INTRODUCTION}

As advances in robotics lend our machines growing prowess, the need to assess their fitness for executing a specified set of tasks within a specified environment grows apace [1]. While some comparison of the length scales of the task and environment have been used to inform the geometry and design of robotic platforms in the past [2] [3], generalizing this concept could prove useful for evaluating the fitness of preexisting robotic architectures, inviting the concepts of 'quasi-static mismatch' and 'dynamic mismatch'. Quasi-static mismatch, the inability of a robot to perform a task in a given environment using quasi-static methods, stems from either geometric constraints or inadequate thermally continuous torque density, while dynamic mismatch arises from insufficient energy or power density. These mismatch concepts could lend useful insight as to why 'generalpurpose' robots (Atlas [4], Big Dog [5], Robo-Simian [6]) designed to navigate and interact within human-scale environments and perform anthropomorphic tasks, are sized at at such scales, as well as suggest opportunities for relaxing the cost and complexity associated with elevated force and length scale architectures in favor of more dynamic behaviors [7].

\section{A. Related Works}

In an effort to better move around within these humanscale environments, some tasks of specific interest in robotics today are those of door opening [8] [9] and stair climbing [10] [11]. Door opening has often been approached by using quasi-static methods with high degree of freedom manipulators, on platforms sized such that they are quasistatically matched to the environment, and the manipulated mechanism lies within the robot's kinematic workspace [12] [13] [14]. While these methods have shown some promise, they are slow, and are either open loop, or require complex control schemes. Furthermore, the sizing of the platform must be matched to the environment such that the kinematic workspace of the platform includes the knob or handle. Stair climbing has been addressed as both a quasi-static, high impedance, inverse kinematic problem [15], as well as a dynamic problem [16] [17] [18]. While all methods have produced results, it is unclear why we prefer a particular method over another with different morphologies and sizings.

\section{B. Contributions}

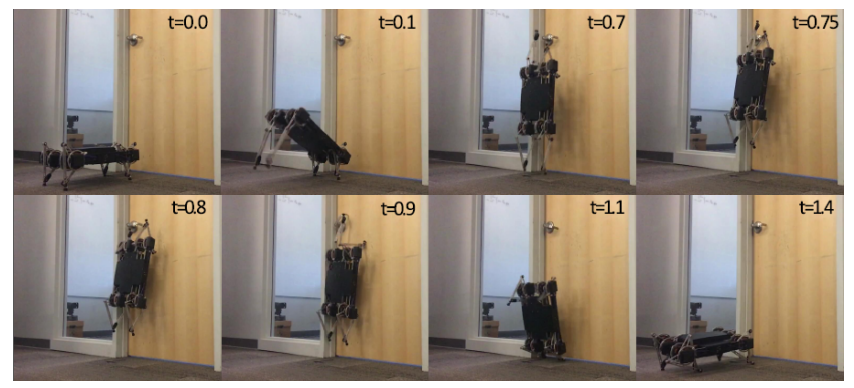

Fig. 1. Sequence of images depicting the dynamic door opening method for Minitaur in a door environment for which it is quasi-statically mismatched.

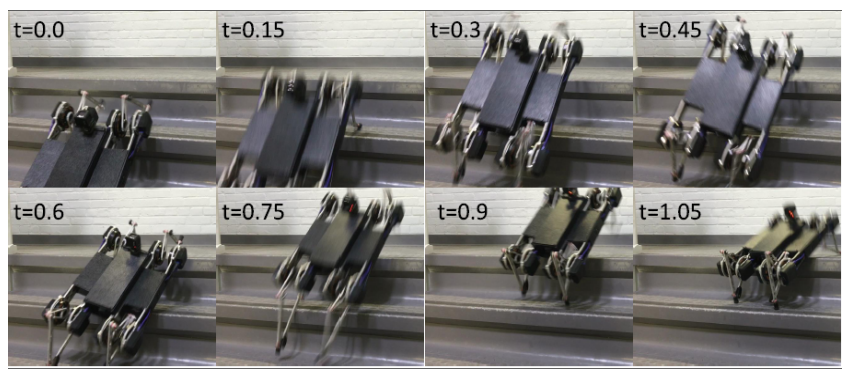

Fig. 2. Sequence of images depicting the dynamic stair climbing method developed for Minitaur on stairs which it is quasi-statically mismatched.

In this paper, we develop a set of necessary conditions for both door opening, outlined in table I, as well as for stair climbing, outlined in table II that determine whether or not a robot can complete each task using only quasi-static maneuvers. This paper considers a representative group of quadrupeds that are less than $10 \mathrm{~kg}$ and are endowed with at least 2 actuated degrees of freedom per leg: Minitaur [19], Little Dog [20], and Super Mini Cheetah [21] proved to be quasi-statically mismatched to one if not both of the examined tasks, yet a simple dynamic analysis of the Minitaur platform as well as subsequent experimental implementations of dynamic methods reveal that completing those tasks is possible in the same environments in which Minitaur was 
TABLE I

NECESSARY CONDITIONS FOR QUASI-STATIC DOOR OPENING ${ }^{1}$

\begin{tabular}{lllll}
\hline Section & Condition & Result & Reason & Eq. \\
\hline III-A.2 & Length-Scale & m,d,s & size \& workspace & $(1)$ \\
III-A.3 & Traction & - & coulomb slip & $(2)$ \\
III-A.4 & Force-Scale & - & door weight & $(3)$ \\
\hline
\end{tabular}

TABLE II

Necessary Conditions For QuAsi-Static Stair Climbing

\begin{tabular}{lllll}
\hline Section & Condition & Result & Reason & Eq. \\
\hline IV-A.1.a & Length-Scale & m,d,s & hip workspace & $(5)$ \\
IV-A.1.b & Torque-Scale & m,d,s & one-leg support & $(6)$ \\
IV-A.2.a & Torque-Scale & m,D,s & limited tread & $(7)$ \\
IV-A.2.b & Traction & M,S & coulomb slip & $(8)$ \\
IV-A.2.c & Torque-Scale & m,S & pinching & $(9)$ \\
\hline
\end{tabular}

We derive conditions necessary ${ }^{2}$ for quasi-statically achieving two useful tasks, thereby proving that three contemporary mesoscale $(1-10 \mathrm{~kg})$ quadrupeds are mismatched to these aspects of their humans'

environments. In contrast, we demonstrate empirically that these tasks can be achieved dynamically at this scale.

quasi-statically mismatched. While the Minitaur platform is physically too short to reach the door handle (1) thereby satisfying none of the quasi-static door opening criteria, it is able to perform a leap, feel for the knob, and push the handle and door simultaneously to open the door, as shown in fig. 1. Similarly, the Minitaur platform has insufficient workspace per (5) and torque density per (6),(7), and (9) to quasi-statically climb human-scale stairs, but it is actually able to climb the stairs by bounding dynamically as shown in fig. 2.

In sum, this paper contributes to our field's still limited understanding of how to characterize a robot's fitness for executing a specified set of tasks within a specified environment [1]. Beyond merely instantiating the insight that smaller, cheaper robots are often capable of performing tasks in human-scale environments using dynamic methods, our necessary conditions bring new rigor to the intuition that these same robots are otherwise mismatched to such tasks when limited to traditional quasi-static strategies. While some of the steps in such a quasi-static analysis may at times seem trivial(e.g. (1)), completing the analysis serves both to quantify intuition (e.g the weight of the door becoming the limiting factor in quasi-static door opening) as well as to reveal in section IV more nuanced criteria bearing on more complex environments such as stairs.

\section{TASK Mismatch}

This paper uses these two representative, arguably useful, tasks to explore the concept of a triple made up of a

\footnotetext{
${ }^{1}$ In the Result column, a capital letter denotes satisfaction and a lower case letter denotes failure for the following robots: 'M/m'-Minitaur, 'D/d'Little, and 'S/s'-Super Mini Cheetah. The absence of any letter denotes that platform was not evaluated against that criterion

${ }^{2}$ We conjecture that the conditions are also sufficient, but proving this goes beyond the scope of the present paper.
}

TABLE III

SUMMARY OF SYMBOLS USED

\begin{tabular}{ll}
\hline Symbol & Description \\
\hline $\mathrm{g}$ & acceleration due to gravity \\
$\mathrm{m}$ & mass of robot \\
$l_{\text {Robot }}$ & full-extension length of robot and limbs \\
$l_{\min }$ & minimum extension of robot limb \\
$l_{\max }$ & maximum extension of robot limb \\
$H$ & height of door knob \\
$R$ & height of stair-riser \\
$T$ & length of stair-tread \\
$\mu$ & environmental coefficient of friction \\
$\theta_{\mathrm{O}}$ & angle at which door is 'open' \\
$\phi_{\mathrm{O}}$ & angle at which handle disengages deadbolt \\
\hline
\end{tabular}

1) task definition

2) environment in which the task is to be completed

3) a robotic architecture that seeks to complete the task.

This abstraction helps categorize particular features of control strategies, as well as reveal necessary conditions bearing on both the environment and the robot architecture for a given triple. For each task, the class of possible quasi-static behaviors available is examined by first restricting the robot to a sagittal plane model, and then using the framework of the Ground Reaction Complex (GRC) [22] to examine the contact modes within the environment that were essential to the completion of each task. Then, optimal physical static configurations representative of these contact modes are used to generate the necessary criteria.

\section{A. Sagittal-Plane Robot Model}

We consider two sagittal plane models of a quadrupedal robot. The first is a true quadruped, the second is a virtual biped where the front and back legs move together. Both assume that only the toes have an arbitrarily large coefficient of friction and the body and limbs have a coefficient of friction of zero. In the quadruped model, the toes may not slide along the surface while any ground reaction force is applied to them. This contrasts with the virtual biped model in which we allow the toes to move along a surface, as long as all configurations that the robot passes through during such movement are statically stable. Thus, quasi-static motion in such a sagittal plane model with only two contacts is still possible.

For both tasks, we examine the parameters of the environment, and impose conditions of mismatch as a function of environmental geometry, and then examine the environmental requirements as well as the platform requirements to produce necessary conditions on quasi-static strategies. Lastly we examine energy requirements for dynamic strategies.

\section{DOOR Opening}

\section{A. Quasi-Static Mismatch Analysis}

The general analysis of the class of quasi-static strategies in door-opening exposes a set of necessary conditions (shown in table I) on robot architectures, and confirms the intuition 
that for real human-scale doors, most smaller, cheaper, quadrupedal platforms will be mismatched, as shown in table IV.

1) Analysis Framework and Environment Model: fig. 3 depicts four distinct contact surfaces, the ground (1), the region of the wall/door excluding the lever-handle (2), the bounded region of the handle (3), or no contact (0). Imposing quasi-static restrictions we discard contact modes $\{2,2\}^{3}$, and $\{3,3\}^{4}$, to conclude that the robot must be in contact mode $\{3,1\}$ to open the door statically. The environment is parametrized such that the door and its handle are torsional springs with spring constants $k_{1}$ and $k_{2}$ respectively, and must be extended to angles $\theta_{O}$ and $\phi_{O}$ respectively to disengage the latch bolt.

2) Length-Scale Necessary Condition: An obvious necessary condition on contact mode $\{3,1\}$ manifests itself as a function of door handle height, $H$, and robot length $l_{\text {robot }}$ :

$$
l_{\text {robot }} \geq H
$$

3) Minimum Environmental Friction: If (1) is satisfied, the derivation in Appendix A shows that the static configuration depicted in fig. 3 requires a minimum allowable coefficient of friction to prevent the toes from slipping. 5

$$
\mu=\frac{k_{1} \theta_{O}}{\operatorname{mg}+k_{2} \phi_{O}}
$$

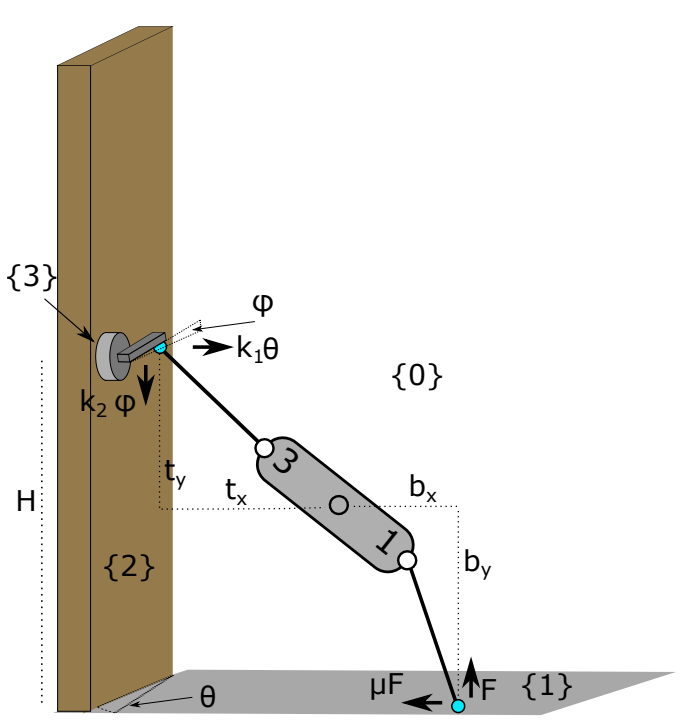

Fig. 3. Forces and Torques on robot in static configuration while opening door

\footnotetext{
${ }^{3}$ If both toes are on the wall, no normal force is being provided, and thus no friction forces are provided to keep the robot static

4 The robot will not be able to push the door ajar without having some contact with surface 1

${ }^{5}$ This constraint is dependent on the assumption that the door handle can not be pushed past the angle $\phi$ which disengages the latch bolt, for as the deflection of the handle exceeds such a position, the required friction to keep the toe on the handle is insufficient.
}

TABLE IV

Evaluation of SELECTED CONTEMPORARy QUAdrupeds AgAinst A STANDARD UNWEIGHTED DOOR

\begin{tabular}{lll}
\hline Robot & $l_{\text {robot }}(m)$ & Handle Height $(\mathrm{m})$ \\
\hline Minitaur & 1 & 1.13 \\
Little Dog & .536 & 1.13 \\
SMC & .7 & 1.13 \\
\hline
\end{tabular}

Each of the robots listed has a body length short enough when compared to the handle height such that it fails condition (1).

4) Minimum Force: Finally, in Appendix B we derive a necessary condition for minimum required force from the balanced torque equations:

$$
F_{\text {req }}=\frac{H k_{1} \theta_{\mathrm{O}}-t_{x} k_{2} \phi_{\mathrm{O}}}{b_{x}}
$$

\section{B. Dynamic Match Analysis}

If indeed the robot is quasi-statically mismatched, it may still be able to use some dynamic method to move, reach, and manipulate the handle and door dynamically.

1) Minimum Energy: We sum the minimum necessary potential energy to reach the knob, the work required to disengage the latch bolt and the work required to push the door sufficiently ajar to derive:

$$
u_{\text {tot }}=\operatorname{mg}\left(H-l_{\text {robot }}\right)+\int_{0}^{\phi_{\mathrm{O}}} k_{2} \phi d \phi+\int_{0}^{\theta_{\mathrm{O}}} k_{1} \theta d \theta
$$

where $l_{\text {robot }}$ is maximum achievable length of the robot.

\section{Experimental Results}

1) Quasi-Static Mismatch and Dynamic Match for Minitaur Platform: Given that international building codes require door handles to be within $0.864 \mathrm{~m}$ and $1.219 \mathrm{~m}$ [23], the necessary condition on the robotic platform having sufficient actuator and/or body length to reach and fully disengage the lever-handle while in a static configuration ${ }^{6}$ will often be infeasible for the Minitaur platform, which has maximum extension of only $1 \mathrm{~m}$. Thus, Minitaur is generally quasistatically mismatched for the task of door opening, and must use dynamic work around methods. The specific door tested had a handle height of $1.13 \mathrm{~m}$, above the meter threshold for quasi-static mismatch on Minitaur. Thus, a dynamic door opening strategy is implemented on the Minitaur platform. A quick analysis of the necessary condition imposed on Minitaur for the dynamic work around shows that each leg in its full range of motion can supply 6.48J [24], and two legs jumping together provide $12.96 \mathrm{~J}$, more than enough energy to satisfy the minimum energy condition of $17 \mathrm{~J}$ for this particular door with handle-lever spring constant of $30.14 \frac{\mathrm{Nm}}{\mathrm{rad}}$, and a door spring constant of $0 \frac{\mathrm{Nm}}{\mathrm{rad}}$.

${ }^{6}\{3,1\}$ in the GRC 
2) Control Strategy: Initial recourse to a purely open loop control strategy starting in contact mode $\{1,1\}$ and pushing off of the hind limbs to invert the platform $\{1,0\}$, and then again pushing off of the fore-limbs to leap with the body in a vertical configuration to put the hind limbs near the handle $\{0,0\}$, and then blindly pushing for the knob with the hind limbs $\{0,3\}$ necessitated trials whose initial conditions were placed at a precise, pre-determined location on the floor in front of the door.

a) Proprioceptive Whisking: While this establishes a proof of concept, reliability of the behavior is greatly improved by augmenting the same open loop procedure to invert and leap, but then rather than using a blind open loop strategy to manipulate the handle, the platform uses the nearest limb as a 'whisker', sweeping the limb through space and using proprioceptive feedback to locate the positions of objects. This technique is reminiscent of rats [25] and has been employed in other robotic systems [26].

3) Results: fig. 4 shows the position of the whisking toe before and after first contact with the handle. Once contact with the handle is perceived using the whisking feedback, the robot retracts the limb a set distance, re-orients it by a pre-determined angular value, and re-extends its $\operatorname{limb}$ to full extension to punch the latch open, all in the span of $200 \mathrm{~ms}$. The use of proprioception to inform the direction of the open loop punching maneuver substantially improves the reliability of the door opening behavior as reported in table V. Despite our reliance on sliding point contact (to overcome the otherwise under-actuated kinematics) using whisked contact to inform the timing and direction of the door opening limb push brings our success rate to $77 \%$, quadrupling that of the blind attempts. Further improvement could come by maintaining contact with the lever-handle after it is found by sweeping the whisking limb. This closedloop feedback might further improve the reliability of this particular dynamic strategy.

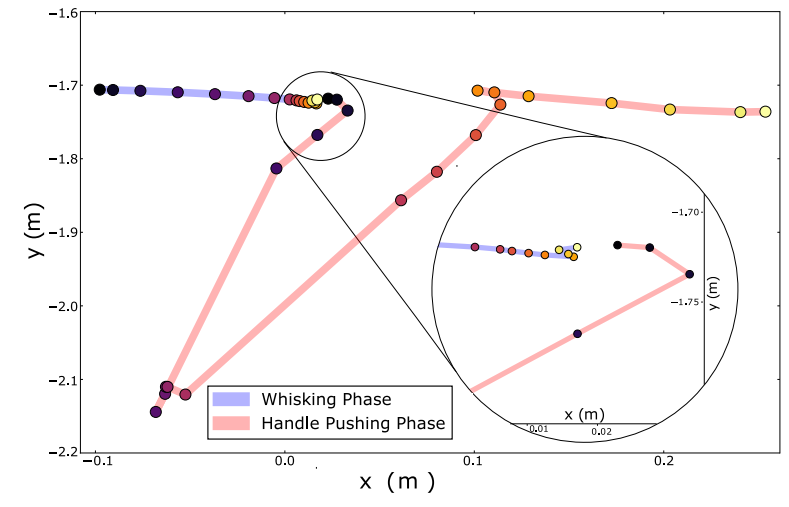

Fig. 4. Position of the whisking toe in Minitaur's COM frame during the time just before it contacts the handle until after it has pushed the handle. The inset area shows the transition from the whisking phase to the the handle pushing phase. Markers are known values at each millisecond, with earlier markers being darker (black represents $t=0$ ). The gradient resets for each subphase.
TABLE V

SuCCESSES OF DYNAMIC DOOR OPENING WITH AND Without PROPRIOCEPTION

\begin{tabular}{lll}
\hline Method & Successes & Failures \\
\hline With Proprioception & 8 & 4 \\
Without Proprioception & 2 & 9 \\
\hline
\end{tabular}

\section{Stair Climbing}

\section{A. Quasi-Static Mismatch Analysis}

Because crawling gaits have proven efficacy in vertical legged ascent [27], we now develop necessary conditions for quasi-static stair climbing match relative to both the sagittal plane quadruped as well as the virtual biped examined in the previous section. Ultimately, as summarized in rows 1-5 of table II we show that the necessary conditions placed on both subclasses of quasi-static strategies are too stringent for the family of smaller, cheaper quadrupeds.

1) Quasi-Static Mismatch for a Sagittal Plane Quadruped Model:

a) Length-Scale Condition: In fig. 5, we ignore the position of the forward hip, and note first that to place one of the hind legs onto the tread of the next stair, the hind hip must be displaced enough from the corner of the step to allow a fully retracted leg with length $l_{\text {min }}$ to pass by. For a step with riser height $R$, this condition is expressed as (5), and if satisfied, no torque limits need be placed on the robot.

$$
R \leq l_{\max }-l_{\min }
$$

b) Minimum Crawl Torque: If (5) is not satisfied, then the lower of the hind toes must be placed some distance from the riser of the stair to ensure the hip is at least distance $l_{\min }$ from the corner of the step. Once one of the hind legs has been lifted, the lower hip is constrained to an annulus centered at the lower toe with radii $l_{\min }$ and $l_{\max }$ until after the recirculated leg is placed on the next tread. Once placed, the toe of the recently placed actuator must bear all of the load of the robot to recirculate the lower toe, and an analysis with this constraint shows that the most favorable configuration is the one depicted in fig. 5. Derived in Appendix C, this places a necessary condition on the robotic platform, and is applied to the platforms in consideration in table VI:

$$
\frac{\tau_{\min }}{\mathrm{m}}=\frac{\mathrm{g}}{2} \sqrt{l_{\min }^{2}-\left(l_{\max }-R\right)^{2}}
$$

2) Quasi-Static Mismatch for a Virtual Biped Model: Returning to the virtual biped model used in the door opening analysis, we again leverage the GRC to examine the stair environment: there are three normal riser surfaces called 1,2 and 3, as well as two tread surfaces, 4 and 5, again with no contact as 0 , as depicted by fig. 6 . We define the task of stair climbing as follows: initially the robot starts in contact mode $\{2,1\}$, and will successfully climb a stair if reaches the contact mode $\{3,2\}$. Of the 36 possible contact modes, we discard reflections, contact modes with no static 


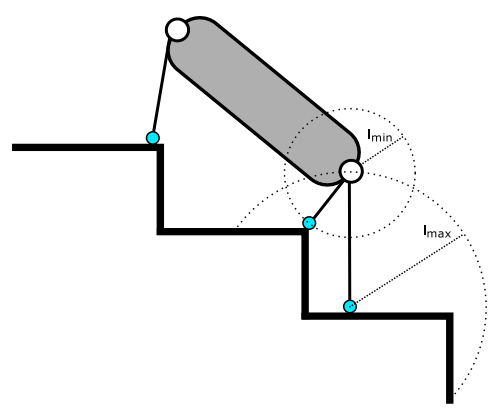

Fig. 5. Sagittal Plane Model of a quadrupedal platform stepping one hind leg to the next stair.

TABLE VI

ANALYSIS OF TORQUE REQUIREMENTS TO PERFORM QUASI STATIC CLIMB WITH A QUADRUPEDAL MODEL

\begin{tabular}{lllcc}
\hline Robot & $\operatorname{mass}(\mathrm{kg})$ & $l_{\min , \max }(\mathrm{m})$ & $\begin{array}{c}I_{\max } \\
\mathrm{m}\end{array}\left(\frac{\mathrm{Nm}}{\mathrm{kg}}\right)$ & $\begin{array}{c}\mathcal{I}_{\text {req }} \\
\mathrm{m}\end{array}\left(\frac{\mathrm{Nm}}{\mathrm{kg}}\right)$ \\
\hline Minitaur & 6 & $0.14,0.28$ & 0.3 & 0.55 \\
Little Dog & 2.85 & $0.075,0.167$ & 0.516 & 4.91 \\
SMC & 9 & $0.1,0.2$ & 0.613 & 4.89
\end{tabular}

This table applies the values in columns 2 and 3 to compute the value in column 5 using condition (6) in section IV-A.1.b, and when compared to column 4 , it shows that all the robots fail to satisfy that requirement. Note that none of the robots satisfy (5) for a standard $R=.196 \mathrm{~m}$ stair.

configurations, a nd $\mathrm{c}$ ontact m odes o nly $\mathrm{r}$ eachable $\mathrm{f}$ rom the goal mode, leaving 11 valid contact modes to examine.

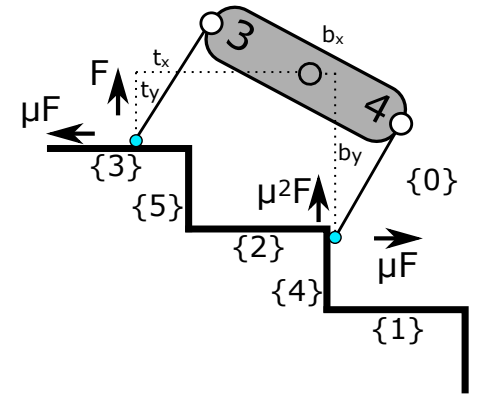

Fig. 6. Stairs Reaction Complex, and Platform in 'Pinching' Configuration with its upper legs on horizontal surface, and its lower legs on a vertical one

There are two valid paths from contact mode $\{2,1\}$ to $\{3,2\}$ within the modified GRC in fig. 7. Thus any successful quasi-static strategies for this model must take one of these two paths, and by examining the contact modes $\{5,2\}$ and $\{3,4\}$ we can impose necessary conditions on the robot architecture such that it has enough mass-specific torque density to maneuver into and out of those contact modes.

a) Single Stair Torque Requirements: When the tread depth is such that contact modes $\{1,1\},\{2,2\}$, and $\{3,3\}$ are not trivial, the robotic platform must assume unfavorable configurations. Again referencing fig. 7, we see that to reach the goal mode, we must pass through contact mode $\{5,2\}$ immediately after contact mode $\{2,2\}$. During this transition, the front toe moves from surface 2 to 5 passing through

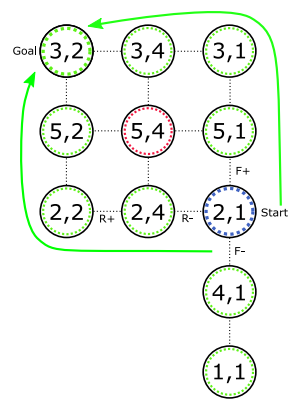

Fig. 7. Valid paths through the GRC from a starting contact mode of $\{2,1\}$ to a target contact mode of $\{3,2\}$

TABLE VII

ANALYSIS OF TORQUE REQUIREMENTS TO UTILIZE CONTACT MODES $\{1,1\},\{2,2\},\{3,3\}$ IN A QUASI-STATIC STRATEGY

\begin{tabular}{|c|c|c|c|c|}
\hline Robot & $\operatorname{mass}(\mathrm{kg})$ & $l_{\mathrm{opt}}(\mathrm{m})$ & $\underset{\mathrm{m}}{\mathcal{I}_{\max }}\left(\frac{\mathrm{Nm}}{\mathrm{kg}}\right)$ & $\underset{\mathrm{m}}{\mathcal{I}_{\text {req }}}\left(\frac{\mathrm{Nm}}{\mathrm{kg}}\right)$ \\
\hline Minitaur & 6 & .14 & 0.6 & 17.5 \\
\hline Little Dog & 2.85 & $\mathrm{n} / \mathrm{a}$ & 1.03 & $\mathrm{n} / \mathrm{a}^{7}$ \\
\hline SMC & 26.5 & .4 & 1.23 & 41.6 \\
\hline
\end{tabular}

Values in columns 2 and 3 are applied to (7) in IV-A.2.a to compute column 5, and when compared with column 4 we note that none of the robots have sufficient mass-specific to rque to ma neuver th rough contact mode $\{5,2\}$.

the corner formed by the two surfaces. Constraining the toe there, a static analysis of the most favorable configuration subject to that constraint provides a necessary torque-scale condition. The most favorable configuration, fig. 8 , is such that the hind toe is at the very edge of the stair, forming two triangles, one right triangle with sides of length $T$ and $l_{\max }$ and a scalar triangle with sides formed by the hypotenuse of the first triangle, the length of the body $l_{b}$, and the extension of the hind leg, $l . \theta$ is derived in Appendix D, and thus the minimum torque can be found by optimizing (7) with respect to $l$ between $l_{\min }$ and $l_{\max }$. table VII shows that such a configuration is unattainable for two of the three platforms.

$$
\underset{\mathrm{m}}{\tau_{\min }}=\frac{\mathrm{gl}}{1+\mu^{2}}(\sin \theta+\mu \cos \theta)
$$

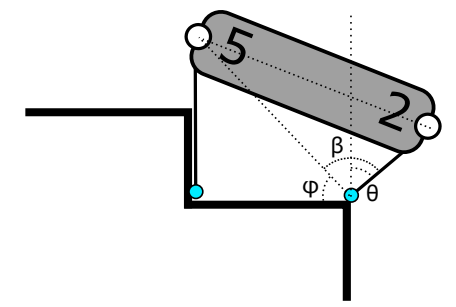

Fig. 8. Analysis of most favorable configuration of virtual bipedal model with contact mode of $\{5,2\}$

\footnotetext{
${ }^{7}$ Because Little Dog is short enough to stand on one tread, the Stair environment analysis we perform here no longer applies; however, a curb climbing analysis should be done and is outside of the scope of this paper.
} 
TABLE VIII

ANALYSIS OF TORQUE REQUIREMENTS TO PERFORM QUASI STATIC Climb as a Virtual Biped Model ${ }^{8}$

\begin{tabular}{lllll}
\hline Robot & $\operatorname{mass}(\mathrm{kg})$ & $l_{\min , \max }(\mathrm{m})$ & $\frac{\tau_{\max }}{\mathrm{m}}\left(\frac{\mathrm{Nm}}{\mathrm{kg}}\right)$ & $\frac{\tau_{\mathrm{req}}}{\mathrm{m}}\left(\frac{\mathrm{Nm}}{\mathrm{kg}}\right)$ \\
\hline Minitaur & 6 & $0.14,0.28$ & 0.6 & 0.77 \\
SMC & 9 & $0.1,0.2$ & 1.23 & 0.77 \\
\hline
\end{tabular}

The values in columns 2 and 3 are applied to (9) in section IV-A.2.c to compute column 5 , and when compared with 4 , we see that only the Super Mini Cheetah has adequate mass-specific torque to perform the pinching maneuver.

b) Pinching Friction Requirement: With the elimination of the contact mode $\{5,2\}$, a robot must then pass through the contact mode $\{3,4\}$. Noting that the robot must transition from contact mode $\{3,1\}$ into $\{3,4\}$, the hind toe can be constrained to the corner of surfaces 1 and 4 . Under this constraint, we find in Appendix E the configuration that requires the lowest environmental coefficient of friction, $\mu$ :

$$
\mu=\frac{-2 R+\sqrt{4 R^{2}+4 b_{x}\left(T-b_{x}\right)}}{2 b_{x}}
$$

c) Pinching Torque Requirement: Assuming sufficient environmental friction, we derive in Appendix $F$ the robotic platform must have sufficient mass-specific torque density:

$$
\frac{\tau_{\text {req }}}{\mathrm{m}}=\frac{R \mathrm{~g} \mu}{\left(1+\mu^{2}\right)}
$$

A survey outlined in table VIII, suggests that this behavior is mismatched for many platforms of this scale.

\section{B. Dynamic Stair Climbing}

As with door opening, the robots evaluated in this paper were quasi-statically mismatched to stair climbing on most human scale staircases. However, as before, the possibility of dynamic solutions depends on the available energy of the platform, and the Minitaur platform proves dynamically matched in this task as well.

1) Analytical Framework: We explore dynamic transitions through the GRC [28], to reach the next stair, and remove the quasi-static constraints placed on the GRC, allowing all contact modes. Furthermore, the starting and goal contact modes are redefined to be the same but periodically shifted up one step, rather than strictly $\{2,1\}$ and $\{3,2\}$ as before.

2) Minimum Energy: This still implies that the COM must move vertically a distance $R$, and this must satisfy:

$$
u_{\min }=\operatorname{mg} R
$$

\section{Experimental Results}

1) Quasi-Static Mismatch and Dynamic Match for Minitaur: The set of stairs tested had $T=.279 \mathrm{~m}$ and $R=.196 \mathrm{~m}$, and Minitaur has $l_{\max }$ and $l_{\min }$ parameters that are effectively $.14 \mathrm{~m}$ and $.28 \mathrm{~m}$ respectively. Minitaur's workspace of $.14 \mathrm{~m}$ is less than the stair rise, failing (5) as outlined in table VI, and subsequently the the required crawling torque is $0.55 \frac{\mathrm{Nm}}{\mathrm{kg}}$, above the single leg continuous limit of $0.3 \frac{\mathrm{Nm}}{\mathrm{kg}}$ [19], failing (6). Furthermore, the single-stair torque limit is $7.08 \frac{\mathrm{Nm}}{\mathrm{kg}}$, and the pinching torque requirement is $0.766 \frac{\mathrm{Nm}}{\mathrm{kg}}$, just above the max continuous torque available by all four motors, $0.6 \frac{\mathrm{Nm}}{\mathrm{kg}}$, failing (7) and (9). Thus, Minitaur is quasi-statically mismatched. However, as the $12.96 \mathrm{~J}$ available in the full range of motion of two of Minitaur's legs [24] is greater than the 11.5248J required in (10) and thus Minitaur is potentially dynamically matched.

2) Strategy and Results: A physical implementation of dynamical stair climbing using the sequence $\{2,1\}-\{1,0\}$ - $\{0,0\}-\{0,2\}-\{3,2\}$ as a strategy was examined. To establish a cyclic bound, the front limbs triggered using proprioceptive information from contact with the next stair to infer when to end one cycle and start another. fig. 9 shows the periodic pitching of the body in parallel with the force experienced by the forelimbs over time. The spikes in force show distinct stairs, which have also been highlighted on the graph as a cycle of the background colors. fig. 10 shows the ground truth $\mathrm{x}$ and $\mathrm{y}$ position of the center of mass as well as body pitch taken from an external 720p 100 fps [29].

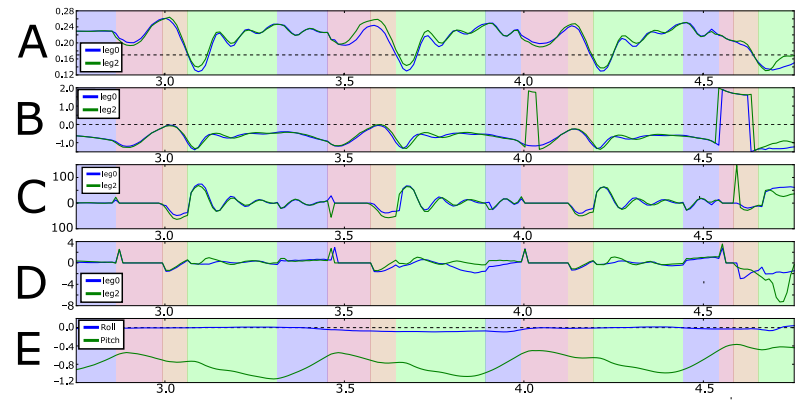

Fig. 9. On-board measurements of pitch and force feedback used to start each cycle of bound from IV-C.2 on sequential steps. (A) depicts leg extension $(m)$, (B) depicts leg angle $(\mathrm{rad})$, (C) depicts radial forces on the legs $(N)$, (D) depicts torque on the legs $(N m)$ and (E) depicts body angle $(\mathrm{rad})$. The changing colors depict subbehaviors within each cycle of the bound

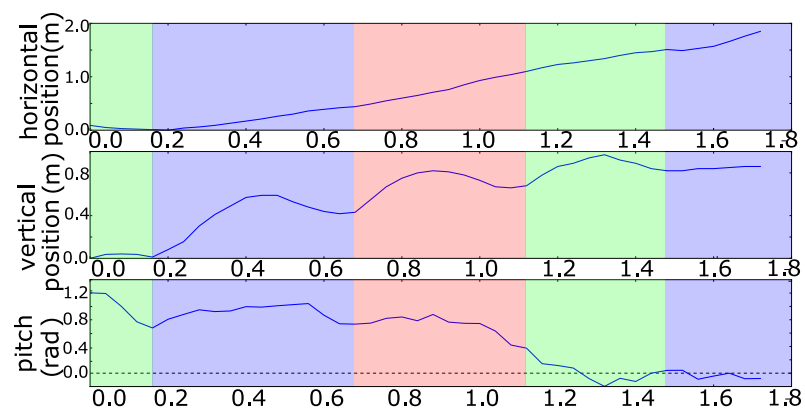

Fig. 10. Ground Truth Tracking of Robot during stair trial showing the cyclic bound strategy described in IV-C.2, where a change in background color indicates the beginning of bound cycle 


\section{CONCLUSION}

This paper addresses the feasibility of robot architectures completing tasks in mismatched environments by establishing the concepts of quasi-static and dynamic mismatch. This quasi-static 'lens' suggests that real world task-environment pairs place such stringent necessary conditions on the space of robot architectures that few, if any, robots can accomplish them. Yet for two useful tasks in such real-world environments, we construct two distinct examples of dynamic solutions that expand the ability of the robot beyond the traditional view of the feasible environment-space.

Work is currently in progress to develop new dynamic solutions to other tasks on the Minitaur platform for which typical environments render quasi-static methods infeasible, such as climbing or descending a chimney or pipe, as well as expanding upon the tasks mentioned in this paper. This will include further improving the reliability of the current solutions by introducing more feedback based control and continuing to leverage the high bandwidth of the direct-drive actuators. Specifically, we will develop a closed-loop control policy for leaping to objects like the door knob, as well as maintaining contact with the door knob while pushing it open, which the authors hope will make the behavior highly repeatable and effecitve.

While quasi-static analysis is often simple and leads to useful and robust control schemes where possible, its failure to do so can instead suggest dynamic analysis of the task in a given enviornment. By attempting to use dynamic solutions in environments where quasi-solutions are infeasible, we can expand the usefulness of any robot architecture, and lessen the need for payloads that accomplish task-specific sensing or actuation - payloads that could impair the robot's ability to accomplish different tasks without modification.

\section{ACKNOWLEDGEMENTS}

This work was supported in part by the ARL/GDRS RCTA project, Coop. Agreement W911NF-1020016 and in part by NSERC (326008481). The authors would also like to thank Avik De for infrastructure support and behavior tuning.

\section{APPENDIX}

\section{A. Derivation of (2):}

For the robot to be statically stable in contact mode $\{3,1\}$, the reaction and friction must cancel, as well as the subsequent torques on the body. In the vertical (Y) direction, we derive:

$$
F=\mathrm{mg}+k_{2} \phi_{\mathrm{O}}
$$

Likewise, the forces in the horizontal direction $(\mathrm{X})$ :

$$
k_{1} \theta_{\mathrm{O}}=\mu F
$$

Substituting $F$ from 11 in 12 gives:

$$
k_{1} \theta_{\mathrm{O}}=\mu\left(\mathrm{mg}+k_{2} \phi_{\mathrm{O}}\right)
$$

And thus the minimum coefficient of friction:

$$
\mu=\frac{k_{1} \theta_{\mathrm{O}}}{\mathrm{mg}+k_{2} \phi_{\mathrm{O}}}
$$

\section{B. Derivation of (3):}

The toe on surface 3 is horizontally displaced by $t_{x}$, and vertically displaced $t_{y}$ from the COM. Similarly the toe on surface 1 is displaced $b_{x}$ and $b_{y}$ from the COM in the $\mathrm{X}$ and $\mathrm{Y}$ directions respectively, and thus the balanced torques on the body must satisfy :

$$
b_{x} F+t_{x} k_{2} \phi_{\mathrm{O}}=b_{y} \mu F+t_{y} k_{1} \theta_{\mathrm{O}}
$$

Substituting for $\mu F$ from 12 in 15 gives:

$$
b_{x}\left(\mathrm{mg}+k_{2} \phi_{\mathrm{O}}\right)+t_{x} k_{2} \phi_{\mathrm{O}}=\left(b_{y}+t_{y}\right) k_{1} \theta_{\mathrm{O}}
$$

Then noting:

$$
H=t_{y}+b_{y}
$$

We substitute and get:

$$
b_{x} F+t_{x} k_{2} \phi_{\mathrm{O}}=H k_{1} \theta_{\mathrm{O}}
$$

Then solving for $\mathrm{F}$ :

$$
F=\frac{H k_{1} \theta_{\mathrm{O}}-t_{x} k_{2} \phi_{\mathrm{O}}}{b_{x}}
$$

C. Derivation of (6):

The angle between the two hind legs is:

$$
\theta=\cos ^{-} 1 \frac{l_{\max }-R}{l_{\min }}
$$

The reaction force on the back toe is at least $\frac{\mathrm{mg}}{2}$, and the thus the torque on the back leg is:

$$
\tau_{\min }=\frac{\mathrm{mg}}{2} l_{\min } \sin \theta
$$

Substituting (20) gives the relation:

$$
\tau_{\min }=\frac{\mathrm{mg}}{2} \sqrt{l_{\min }^{2}-\left(l_{\max }-R\right)^{2}}
$$

D. Derivation of (7):

From fig. 8 , we calculate $\beta$ and $\phi$ to find $\theta$ :

$$
\begin{gathered}
\phi=\tan ^{-} 1\left(\frac{l_{\max }}{T}\right) \\
\beta=\cos ^{-} 1\left(\frac{l^{2}-l_{b}^{2}+l_{\max }^{2}+T^{2}}{2 l \sqrt{l_{\max }^{2}+T^{2}}}\right)
\end{gathered}
$$

And then $\theta$ is simply:

$$
\theta=\beta+\phi-\frac{\pi}{2}
$$

\section{E. Derivation of (8):}

Let the reaction force on the front toe be $F$, and the friction force be $\mu F$. Then, for static stability the reaction force on the rear toe must be $\mu F$ and the friction force $\mu^{2} F$. Let the front toe be displaced horizontally and vertically from the COM by $t_{x}$ and $t_{y}$ respectively, and let the hind toe be similarly displaced by $b_{x}$ and $b_{y}$. Thus the following balanced torque equation can be written 9 :

$$
t_{x} F+b_{y} \mu F=b_{x} \mu^{2} F+t_{y} \mu F
$$

${ }^{9}$ If the COM is below surface 3 , then the $t_{x}$ term will on the opposite side of the equation; however, $2 R=b_{y}+t_{y}$ in this case, and so the result in (28) will be the same. 
Canceling $F$ and solving for $\mu$ yields

$$
\mu=\frac{-\left(b_{y}-t_{y}\right)_{-}^{+} \sqrt{\left(b_{y}-t_{y}\right)^{2}+4 b_{x} t_{x}}}{2 b_{x}}
$$

Finally, we note that $b_{y}-t_{y}=2 R$, and that the coefficient of friction must be positive, yielding:

$$
\mu=\frac{-2 R+\sqrt{4 R^{2}+4 b_{x} t_{x}}}{2 b_{x}}
$$

Lastly, to minimize $\mu$, we maximize $b_{x}$, and minimize $t_{x}$, which imposes the constraint $T=b_{x}+t_{x}$ :

$$
\mu=\frac{-2 R+\sqrt{4 R^{2}+4 b_{x}\left(T-b_{x}\right)}}{2 b_{x}}
$$

\section{F. Derivation of (9):}

From Appendix E, we then balance the vertical forces:

$$
F=\frac{\mathrm{mg}}{\left(1+\mu^{2}\right)}
$$

Noting that the hind leg must be extended at least $R$ to allow the body to be angled toward the next stair, we notice that the torque on the hind legs must be:

$$
\tau=R \frac{\mathrm{mg}}{\left(1+\mu^{2}\right)}
$$

\section{REFERENCES}

[1] D. E. Kodistchek, V. Kumar, and D. D. Lee, Future Directions of Intelligent Physical Systems, ser. Future Directions Workshops, Oct 2015. [Online]. Available: http://www.acq.osd.mil/rd/basic_research/ references/docs/4_FISAL.pdf 1, 2

[2] L. Stocco, S. Salcudean, and F. Sassani, "On the use of scaling matrices for task-specific robot design," IEEE Transactions on Robotics and Automation, vol. 15, no. 5, pp. 958-965, 1999. 1

[3] M. Tandirci, J. Angeles, and F. Ranjbaran, "Characteristic point and the characteristic length of robotic manipulators," vol. 45, 1992, pp. 203-208. 1

[4] G. Nelson, A. Saunders, N. Neville, B. Swilling, J. Bondaryk, D. Billings, C. Lee, R. Playter, and M. Raibert, "Petman: A humanoid robot for testing chemical protective clothing," $t$, vol. 30 , no. 4 , pp. 372-377, 2012. 1

[5] M. Raibert, K. Blankespoor, G. Nelson, and R. Playter, "Bigdog, the rough-terrain quaduped robot," 2008. 1

[6] P. e. a. Herbert, "Mobile manipulation and mobility as manipulation - design and algorithms of robosimian," Journal of Field Robotics, vol. 32, no. 2, pp. 255-274, 2015.1

[7] B. D. Miller and J. E. Clark, "Dynamic similarity and scaling for the design of dynamical legged robots," in Intelligent Robots and Systems (IROS), 2015 IEEE/RSJ International Conference on, Sept 2015, pp. 5719-5726. 1

[8] N. Banerjee, X. Long, R. Du, F. Polido, S. Feng, Atkeson, C. G., M. Gennert, and T. Padir, "Human-supervised control of the atlas humanoid robot for traversing doors," Humanoid Robots, IEE RAS, vol. 1, pp. 722-729, 2015. 1

[9] H. Arisumi, J. Chardonnet, and K. Yokoi, "Whole-body motion of a humanoid robot for passing through a door - opening a door by impulsive force," nternational Conference on Intelligent Robots and Systems, pp. 428-434, 2009. 1

[10] C. Fu and K. Chen, "Gait synthesis and sensory control of stair climbing for a humanoid robot," IEEE Transactions on Industrial Electronics, vol. 55, no. 5, pp. 2111-2120, 2008. 1

[11] B. Seo, H. Kim, M. Kim, K. Jeong, and T. Seo, "Flipbot: A new field robotic platform for fast stair climbing," International Journal of Precision Engineering and Manufacturing, vol. 14, no. 11, pp. 19091914, 2013. 1

[12] W. Chung, C. Rhee, Y. Shim, H. Lee, and S. Park, "Door-opening control of a service robot using the multifingered robot hand," IEEE Transactions on Industrial Electronics, vol. 56, 2009. 1
[13] C. Rhee, W. Chung, K. Munsang, Y. Shim, and H. Lee, "Door opening control using the multi-fingered robotic hand for the indoor service robot," Robotics and Automation, 2004. Proceedings. ICRA '04. 2004 IEEE International Conference on, pp. 4011-4016, 2004. 1

[14] K. Nagatani and S. Yuta, "Designing a behavior to open a door and to pass through a door-way using a mobile robot equipped with a manipulator," International COnference on Intelligent Robots and Systems, vol. 2, pp. 847-853, 1994. 1

[15] C.-L. Shih, "Ascending and descending stairs for a biped robot," Transactions On Systems, Man, and Cyberneteics, pp. 255-268, 1999. 1

[16] S. A. Stoeter and N. Papanikolopoulos, "Autonomous stair-climbing with miniature jumping robots," IEEE TRANSACTIONS ON SYS TEMS, MAN, AND CYBERNETICS, no. pt 2, pp. 313-325, 2005. 1

[17] E. Moore, D. Campbell, F. Grimminger, and M. Buehler, "Reliable stair climbing in the simple hexapod 'rhex'," Robotics and Automation, pp. 2222-2227, 2002. 1

[18] S. Talebi, M. Buehler, and E. Papadopoulos, "Towards Dynamic Step Climbing For A Quadruped Robot with Compliant Legs," IEEE/RSJ International Conference on Intelligent Robots and Systems (In Press), 2000. 1

[19] G. Kenneally, A. De, and D. Koditschek, "Design principles for a family of direct-drive legged robots," IEEE Robotics and Automation Letters, vol. PP, no. 99, pp. 1-1, 2016. 1, 6

[20] A. Shkolnik, M. Levashov, I. R. Manchester, and R. Tedrake, "Bounding on rough terrain with the LittleDog robot," The International Journal of Robotics Research, vol. 30, no. 2, pp. 192-215, Feb. 2011. [Online]. Available: http://ijr.sagepub.com/cgi/ doi/10.1177/0278364910388315 1

[21] W. Bosworth, S. Kim, and N. Hogan, "The mit super mini cheetah: A small, low-cost quadrupedal robot for dynamic locomotion," 2015, cited By 0. [Online]. Available: https: //www.scopus.com/inward/record.uri?eid=2-s2.0-84967262690\& partnerID $=40 \& m d 5=5 b 305920 \mathrm{~d} 0 \mathrm{cc} 72 \mathrm{~b} 789487 \mathrm{dc} 49595 \mathrm{~d} 18 \mathrm{a} 1$

[22] A. Johnson and D. E. Koditschek, "Toward a vocabulary of legged leaping," c. IEEE Intl. Conf. Robotics and Automation, 2013. 2

[23] International Code Council, "Accessible and usable buildings and facilities," vol. 1008.1.9.2, no. A117.1, 2009. 3

[24] G. Kenneally and D. E. Koditschek, "Leg Design for Energy Management in an Electromechanical Robot," IEEE/RSJ International Conference on Intelligent Robots and Systems (In Press), 2015. 3, 6

[25] D. Kleinfeld, E. Ahissar, and M. Diamond, "Active sensation: insights from the rodent vibrissa sensorimotor system," Current Opinion in Neurobiology, vol. 16, no. 4, pp. 435-444, 2006. 4

[26] T. Prescott, M. Pearson, B. Mitchinson, J. Sullivan, and A. Pipe "Whisking with robots: From rat vibrissae to biomimetic technology for active touch," IEEE Robotics and Automation Magazine, vol. 16, no. 3, pp. 42-50, 2009. 4

[27] G. C. Haynes, "Gait regulation control techniques for robust legged locomotion," 2008. 4

[28] A. L. Brill, A. De, A. M. Johnson, and D. E. Koditschek, "Tailassisted rigid and compliant legged leaping," IEEE/RSJ International Conference on Intelligent Robots and Systems, p. (in press). 6

[29] GoPro. [Online]. Available: http://shop.gopro.com/cameras/ hero-session/CHDHS-102.html 6 